\title{
Clinical and cytogenetic diversity in Fanconi's anaemia
}

\author{
G DUCKWORTH-RYSIECKI*, M HULTÉN†, J MANNł, AND A M R TAYLOR* \\ From * the Department of Cancer Studies, Cancer Research Campaign Laboratories, University of \\ Birmingham Medical School, Birmingham B15 2TJ; †the Regional Cytogenetics Laboratory, East \\ Birmingham Hospital, Bordesley Green East, Birmingham B9 5ST; and $\ddagger$ the Birmingham Children’s \\ Hospital, Ladywood Middleway, Ladywood, Birmingham B16.
}

SUMMARY Abnormally high levels of spontaneous and mitomycin $\mathbf{C}$ or diepoxybutane induced chromosome breakage were observed in lymphocytes from eight out of nine previously undescribed patients clinically diagnosed as having Fanconi's anaemia. The results suggest that the combination of spontaneous and induced chromosome breakage is a good aid in the differential diagnosis and we suggest that increased chromosome breakage is pathognomonic for this recessive disorder. It is, however, not possible to demonstrate consistently raised levels of induced chromosome breakage in obligate carriers. The patient who had normal levels of chromosome breakage had an atypical haematological picture and may suffer from a disease genetically different from Fanconi's anaemia.

Fanconi's anaemia is an autosomal recessive disorder in which patients develop hypoplastic anaemia. It is usually associated with diverse congenital abnormalities which may include skeletal deformities, skin hyperpigmentation, growth retardation, deafness, and genital, ocular, renal, and cardiac defects. ${ }^{1}$ Patients also have an abnormally high risk of developing malignant disease, particularly acute myelomonocytic leukaemia. ${ }^{2}$

One of the most striking features of Fanconi's anaemia is the variation between symptoms and the course of the disease in different patients. Such variation may occur among unrelated cases, but also between sibs. ${ }^{34}$ Patients also show wide variation in the age of onset of haematological symptoms and in the course of the disease. ${ }^{56}$ Such variation, particularly in the absence of congenital abnormalities or other affected sibs, can lead to difficulties in the diagnosis. ${ }^{7}$

Many authors have shown that cultured lymphocytes from Fanconi's anaemia patients display abnormally high levels of chromosome breakage, both spontaneously and following treatment with bifunctional alkylating agents such as mitomycin $\mathrm{C}^{8}$ (MMC) or diepoxybutane (DEB). ${ }^{9}$ These features have been used extensively as aids in the

Received for publication 5 October 1983. Accepted for publication 10 October 1983. diagnosis of Fanconi's anaemia. However, some authors claim that certain patients with typical clinical manifestations of Fanconi's anaemia show normal levels of spontaneous and induced lymphocyte chromosome breakage. ${ }^{\mathbf{4 1 0}}$ Conversely, abnormally high levels of spontaneous and induced chromosome breakage have been shown in some patients not judged to have the necessary clinical symptoms for a diagnosis of the disease. ${ }^{7}$ All these findings suggest that Fanconi's anaemia may be heterogeneous with regard to chromosomal instability. However, since the diagnosis is complicated by both variation in clinical symptoms and the existence of similar symptoms in some other disorders, great care is obviously necessary when diagnosing the disease in cytogenetically normal patients.

In the present study lymphocytes from nine previously unreported patients with Fanconi's anaemia, ascertained because of their clinical symptoms, were compared in order to investigate whether there was any consistent inter-patient variation in the levels of spontaneous and induced chromosome breakage. The usefulness of cytogenetic methods in the diagnosis of the disease and in the detection of heterozygotes is discussed.

\section{Materials}

Blood samples were obtained from nine unrelated 
TABLE 1 Summary of details of Fanconi's anaemia patients.

\begin{tabular}{|c|c|c|c|c|c|}
\hline & \multicolumn{5}{|l|}{ Subject } \\
\hline & $F A 2 B I$ & $F A 3 B I$ & $F A S B I$ & $F A 6 B I$ & $F A 7 B I$ \\
\hline $\begin{array}{l}\text { Year of birth } \\
\text { Sex } \\
\text { Congenital } \\
\text { abnormalities }\end{array}$ & $\begin{array}{l}1953 \\
\text { M } \\
\text { Oesophageal atresia } \\
\text { Accessory thumb } \\
\text { Systolic murmur }\end{array}$ & $\begin{array}{l}1971 \\
\text { F } \\
\text { Microcephaly } \\
\text { Small kidney } \\
\text { Bilateral ureteric } \\
\quad \text { reflux }\end{array}$ & $\begin{array}{l}1957 \\
\text { F } \\
\text { Duodenal obstruction } \\
\text { Skin hyperpigmentation }\end{array}$ & $\begin{array}{l}1967 \\
\text { M } \\
\text { Skin hyperpigmentation } \\
\text { Systolic murmur }\end{array}$ & $\begin{array}{l}1969 \\
\text { F } \\
\text { Absent thumb } \\
\text { Bilateral ureteric } \\
\text { reflux } \\
\text { Skin } \\
\text { hyperpigmentation }\end{array}$ \\
\hline $\begin{array}{l}\text { Age of onset of } \\
\text { haematological } \\
\text { changes }\end{array}$ & 11 & 8 & 12 & 12 & 4 \\
\hline $\begin{array}{l}\text { Representative } \\
\text { blood counts } \\
\text { before treatment }\end{array}$ & $\begin{array}{l}\text { Hb } 6.4 \mathrm{~g} / \mathrm{dl} \\
\text { WCC } 2.7 \times 10^{9} / 1 \\
\text { Platelets } 1 \times 10^{9} / 1\end{array}$ & $\begin{array}{l}\mathrm{Hb} 9 \cdot 2 \mathrm{~g} / \mathrm{dl} \\
\text { WCC } 3 \cdot 2 \times 109 / 1 \\
\text { Platelets } 30 \times 10^{9} / 1\end{array}$ & $\begin{array}{l}\text { Hb } 10-11 \mathrm{~g} / \mathrm{dl} \\
\text { WCC } 2-3 \times 10^{9} / 1 \\
\text { Platelets } 20-40 \times 10^{9} / 1\end{array}$ & $\begin{array}{l}\mathrm{Hb} 7.5 \mathrm{~g} / \mathrm{dl} \\
\text { WCC } 3.8 \times 10^{9} / 1 \\
\text { Platelets } 50 \times 10^{9} / 1\end{array}$ & $\begin{array}{l}\text { Hb 9-10 g/dl } \\
\text { WCC 5-13 } \times 109 / 1 \\
\text { Platelets } 8-25 \times 109 / 1\end{array}$ \\
\hline $\begin{array}{l}\text { Height } \\
\text { Weight }\end{array}$ & $\begin{array}{l}<3 \text { rd centile } \\
<3 \text { rd centile }\end{array}$ & $\begin{array}{l}\text { Normal } \\
\text { Normal }\end{array}$ & $\begin{array}{l}<3 \text { rd centile } \\
<3 \text { rd centile }\end{array}$ & $\begin{array}{l}\text { 10th centile } \\
<3 \text { rd centile }\end{array}$ & $\begin{array}{l}\text { 3rd centile } \\
\text { 3rd centile }\end{array}$ \\
\hline Treatment & $\begin{array}{l}\text { Prednisone } \\
\text { Splenectomy } \\
\text { Oxymetholone } \\
\text { Blood transfusion }\end{array}$ & Oxymetholone & $\begin{array}{l}\text { Prednisone } \\
\text { Oxymetholone } \\
\text { Thyroxine }\end{array}$ & None & $\begin{array}{l}\text { Blood transfusion } \\
\text { Oxymetholone }\end{array}$ \\
\hline Other & $\begin{array}{l}\text { Dystrophic nails } \\
\text { Tachycardia }\end{array}$ & None & $\begin{array}{l}\text { Three sibs died } \\
\text { neonatally with } \\
\text { congenital heart } \\
\text { disease } \\
\text { Hypothyroidism }\end{array}$ & None & $\begin{array}{l}\text { Bone age retarded } \\
\text { Deafness } \\
\text { Raised IgA }\end{array}$ \\
\hline
\end{tabular}

patients diagnosed on clinical grounds as having Fanconi's anaemia and showing a variety of clinical phenotypes (table 1). In addition, samples of blood were obtained from a patient who had no haematological abnormality, but was short in stature and was initially referred as a possible case of Turner's syndrome (miscellaneous blood-MB8) (table 1). Samples were also taken from three Fanconi's anaemia heterozygotes (FAHET3 and FAHET8 are the mothers of FA3BI and FA8BI, respectively; FAHET5 is the father of FA5BI). Control blood samples were obtained from nine normal healthy subjects, four male and five female, aged between 21 and 32.

\section{Methods}

Heparinised venous blood samples were cultured in duplicate by adding $0.5 \mathrm{ml}$ whole blood to $4.5 \mathrm{ml}$ Ham's F10 medium (Flow Laboratories Ltd), containing $10 \%$ bovine serum and $0.05 \mathrm{ml}$ phytohaemagglutinin (PHA, Burroughs-Wellcome Ltd). Colchicine was added for the last hour in a 48 or 72 hour culture. Cells were treated with hypotonic potassium chloride solution $(0.075 \mathrm{~mol} / \mathrm{l})$, fixed in methanol:acetic acid (3:1), air dried, and stained in $2 \%$ orcein. Slides were coded and metaphases scored for all types of chromosome damage, according to ISCN nomenclature. ${ }^{11}$

\section{MITOMYCIN C TREATMENT}

Mitomycin C (MMC, Sigma), obtained in crystalline form in vials containing $2 \mathrm{mg}$, was reconstituted by the addition of $4 \mathrm{ml}$ sterile glass distilled water. Lymphocytes were treated immediately before culture at the $G_{0}$ stage of the cell cycle. A total of $0.5 \mathrm{ml}$ whole blood was added to $4.5 \mathrm{ml}$ Ham's F10 medium containing $10 \%$ bovine serum and MMC, giving a final MMC concentration of $0 \cdot 1,0.25$, or $0.5 \mu \mathrm{g} \mathrm{ml}^{-1}$. The blood was incubated in the dark at $37^{\circ} \mathrm{C}$ for 30 minutes before being centrifuged at $500 \mathrm{~g}$ for 5 minutes and washed twice in $5 \mathrm{ml}$ Ham's F10 medium. It was then cultured as described above and harvested after 48 hours.

\section{DIEPOXYBUTANE TREATMENT}

Diepoxybutane (DEB, Aldrich) was added to blood cultures 24 hours after PHA stimulation to give a final concentration of 0.01 or $0.1 \mu \mathrm{g} \mathrm{ml}^{-1}$. Cultures were harvested after 72 hours.

\section{Results}

SPONTANEOUS ABERRATIONS

Analysis of 1150 cells from nine normal subjects revealed very low levels of spontaneous chromosome breakage (mean of 1.8 aberrations per 50 cells, table 2), the majority of aberrations being chromatid 


\begin{tabular}{|c|c|c|c|c|}
\hline$F A 8 B I$ & $F A 9 B I$ & $F A 1 O B I$ & $F A I I B I$ & $M B 8$ \\
\hline $\begin{array}{l}1977 \\
\text { F } \\
\text { Absent radius and thumb } \\
\text { Abnormal thumb } \\
\text { Persistent ductus arteriosus } \\
\text { Flattened auricle } \\
\text { Microcephaly } \\
\text { Slight skin hyperpigmentation } \\
\text { Microphthalmia } \\
\text { Single kidney }\end{array}$ & $\begin{array}{l}1969 \\
\text { M } \\
\text { Abnormal displaced thumbs } \\
\text { Absent radial pulses } \\
\text { Systolic heart murmur } \\
\text { Skin hyperpigmentation } \\
\text { Abnormal urethra }\end{array}$ & $\begin{array}{l}1978 \\
\text { M } \\
\text { Short radius } \\
\text { Abnormal thumb }\end{array}$ & $\begin{array}{l}1978 \\
\text { M } \\
\text { Strabismus } \\
\text { Small eyes } \\
\text { Microcephaly }\end{array}$ & $\begin{array}{l}1966 \\
\text { F } \\
\text { Strabismus } \\
\text { Ptosis right eye } \\
\text { Systolic heart murmur }\end{array}$ \\
\hline 3 & $6 \frac{1}{2}$ & $<1$ & 4 & None \\
\hline $\begin{array}{l}\text { Hb } 11 \cdot 5 \mathrm{~g} / \mathrm{dl} \\
\text { WCC } 12 \cdot 2 \times 10^{9} / 1\end{array}$ & $\begin{array}{l}\text { Hb } 9.2 \mathrm{~g} / \mathrm{dl} \\
\text { WCC } 4.7 \times 10^{9} / 1 \\
\text { Platelets } 36 \times 10^{9} / 1\end{array}$ & $\begin{array}{l}\text { Hb } 2-9 \mathrm{~g} / \mathrm{dl} \\
\text { WCC } 2-9 \times 10^{9} / 1 \\
\text { Platelets normal }\end{array}$ & $\begin{array}{l}\mathrm{Hb} 8 \cdot 1 \mathrm{~g} / \mathrm{dl} \\
\text { WCC } 3 \cdot 9 \times 10^{9} / 1 \\
\text { Platelets } 26 \times 10^{9} / 1\end{array}$ & $\begin{array}{l}\text { Hb } 13.5 \mathrm{~g} / \mathrm{dl} \\
\text { WCC } 5.5 \times 10^{9} / 1\end{array}$ \\
\hline Small & $\begin{array}{l}\text { 3rd centile } \\
<10 \text { th centile }\end{array}$ & $\begin{array}{l}<3 \text { rd centile } \\
<3 \text { rd centile }\end{array}$ & Small & $<3$ rd centile \\
\hline None & $\begin{array}{l}\text { Blood transfusion } \\
\text { Oxymetholone } \\
\text { Prednisone }\end{array}$ & $\begin{array}{l}\text { Corticosteroids } \\
\text { Androgens } \\
\text { Blood transfusion }\end{array}$ & None & None \\
\hline Birth weight $2.07 \mathrm{~kg}$ & $\begin{array}{l}\text { Birth weight } 2 \cdot 27 \mathrm{~kg} \\
1 \text { sib died neonatally with } \\
\text { congenital heart disease } \\
\text { Low bone age }\end{array}$ & Parents first cousins & $\begin{array}{l}\text { Birth weight } 2 \cdot 10 \mathrm{~kg} \\
\text { Intrapartum asphyxia } \\
\text { Neonatal jaundice } \\
\text { Hyperactive }\end{array}$ & \\
\hline
\end{tabular}

gaps and breaks. Low levels of spontaneous chromosome breakage were also observed in lymphocytes from three obligate heterozygotes (mean of 2.33 aberrations per 50 cells, table 2 ).

Abnormally high levels of spontaneous chromosome breakage (chromatid gaps, breaks, and interchanges) were observed in lymphocytes from six of the nine clinically diagnosed Fanconi's anaemia patients studied (range of 18 to 39 aberrations per 50 cells, mean of $26 \cdot 0$, table 2 ). However, lymphocytes from FA3BI, and especially FA11BI, showed lower rates of spontaneous chromosome breakage, while in lymphocytes from one apparent case of Fanconi's anaemia (FA10BI) no spontaneous chromosome aberrations were present. Independent blood samples from individual patients gave quantitatively consistent results (table 2), but levels of chromosome breakage did vary between the different patients.

Lymphocytes from patient MB8, who has short stature but no haematological symptoms, when cultured on two separate occasions (table 2) also showed a very high rate of spontaneous chromosome breakage with 31 and 29 aberrations per 50 cells, respectively. The rate and pattern of chromosome breakage in this patient were clearly abnormal and fell within the range found for patients with Fanconi's anaemia.
INDUCED ABERRATIONS

In order to calculate the number of chromosome aberrations induced by MMC or DEB, the total number of spontaneous aberrations scored in 50 cells from each person was subtracted from the total number of aberrations scored in 50 treated cells. $\mathrm{G}_{0}$ MMC treatment induced low levels of chromosome aberrations in lymphocytes from seven normal subjects. Treatment with $0 \cdot 1,0 \cdot 25$, or $0.5 \mu \mathrm{g} \mathrm{ml}^{-1}$ MMC resulted in the induction of 0 to 4 (mean $0 \cdot 58$ ), 0 to 5 (mean $2 \cdot 0$ ), and 0 to 17 (mean 5.2) aberrations per 50 cells respectively (tables 3 and 4 ). The majority of induced aberrations were chromatid gaps and breaks with occasional quadriradials. Similar results were obtained with lymphocytes from three Fanconi's anaemia heterozygotes (tables 3 and 4). Treatment of Fanconi's anaemia lymphocytes with $0 \cdot 1,0.25$, or $0.5 \mu \mathrm{g} \mathrm{ml}^{-1} \mathrm{MMC}$ resulted in the induction of 19 to 133,47 to 100 , and 112 to 265 aberrations per 50 cells respectively, much higher than levels in normal subjects or heterozygotes (tables 3 and 4). No induced aberrations were observed in lymphocytes from FA10BI following treatment with $0.5 \mu \mathrm{g} \mathrm{ml}^{-1} \mathrm{MMC}$ (table 4). Lymphocytes from patient MB8 contained highly abnormal levels of induced chromosome aberrations following treatment with $0 \cdot 1 \mu \mathrm{g} \mathrm{ml}^{-1} \mathrm{MMC}$ on two separate occasions (table 3 ). 
TABLE 2 Spontaneous chromosome breakage in lymphocytes from Fanconi's anaemia patients, MB8, heterozygotes, and normal controls.

\begin{tabular}{|c|c|c|c|c|c|c|c|c|c|c|c|}
\hline \multirow[t]{2}{*}{ Subject } & \multirow{2}{*}{$\begin{array}{l}\text { No of cells } \\
\text { analysed }\end{array}$} & \multicolumn{8}{|c|}{ No of } & \multirow{2}{*}{$\begin{array}{l}\text { Total No of } \\
\text { aberrations }\end{array}$} & \multirow{2}{*}{$\begin{array}{l}\% \text { aberrant } \\
\text { cells }\end{array}$} \\
\hline & & $r$ & dic & $f$ & $c t g$ & $c t b$ & csg & $q r$ & $t r$ & & \\
\hline FA2BI & 50 & 0 & 1 & 3 & 5 & 6 & 0 & 5 & 0 & 20 & 22 \\
\hline FA3BI & $\begin{array}{l}50 \\
50 \\
50 \\
50\end{array}$ & $\begin{array}{l}0 \\
0 \\
0 \\
0\end{array}$ & $\begin{array}{l}\mathbf{0} \\
\mathbf{0} \\
\mathbf{0} \\
\mathbf{0}\end{array}$ & $\begin{array}{l}2 \\
2 \\
0 \\
0\end{array}$ & $\begin{array}{l}8 \\
5 \\
4 \\
9\end{array}$ & $\begin{array}{l}2 \\
3 \\
2 \\
3\end{array}$ & $\begin{array}{l}2 \\
0 \\
0 \\
0\end{array}$ & $\begin{array}{l}1 \\
0 \\
0 \\
0\end{array}$ & $\begin{array}{l}0 \\
0 \\
0 \\
0\end{array}$ & $\begin{array}{r}15 \\
10 \\
6 \\
12\end{array}$ & $\begin{array}{l}22 \\
18 \\
12 \\
22\end{array}$ \\
\hline FA5BI & 50 & 0 & 1 & 1 & 5 & 9 & 3 & 2 & 2 & 23 & 32 \\
\hline FA6BI & $\begin{array}{l}50 \\
50\end{array}$ & $\begin{array}{l}\mathbf{0} \\
\mathbf{0}\end{array}$ & $\begin{array}{l}\mathbf{0} \\
\mathbf{0}\end{array}$ & $\begin{array}{l}1 \\
2\end{array}$ & $\begin{array}{r}6 \\
10\end{array}$ & $\begin{array}{r}10 \\
8\end{array}$ & $\begin{array}{l}0 \\
0\end{array}$ & $\begin{array}{l}0 \\
0\end{array}$ & $\begin{array}{l}1 \\
0\end{array}$ & $\begin{array}{l}18 \\
20\end{array}$ & $\begin{array}{l}24 \\
30\end{array}$ \\
\hline FA7BI & 50 & 0 & 0 & 2 & 19 & 5 & 0 & 0 & 1 & 27 & 40 \\
\hline FA8BI & 50 & 0 & 0 & 0 & 15 & 8 & 0 & 3 & 0 & 26 & 34 \\
\hline FA9BI & $\begin{array}{l}50 \\
50 \\
50\end{array}$ & $\begin{array}{l}0 \\
0 \\
0\end{array}$ & $\begin{array}{l}0 \\
1 \\
1\end{array}$ & $\begin{array}{l}2 \\
0 \\
4\end{array}$ & $\begin{array}{l}17 \\
19 \\
29\end{array}$ & $\begin{array}{r}7 \\
11 \\
5\end{array}$ & $\begin{array}{l}1 \\
0 \\
0\end{array}$ & $\begin{array}{l}\mathbf{0} \\
\mathbf{3} \\
\mathbf{0}\end{array}$ & $\begin{array}{l}0 \\
0 \\
0\end{array}$ & $\begin{array}{l}27 \\
34 \\
39\end{array}$ & $\begin{array}{l}36 \\
42 \\
52\end{array}$ \\
\hline FA10BI & 22 & 0 & 0 & 0 & 0 & 0 & 0 & 0 & 0 & 0 & 0 \\
\hline FA11BI & $\begin{array}{l}50 \\
50\end{array}$ & $\begin{array}{l}0 \\
0\end{array}$ & $\begin{array}{l}0 \\
0\end{array}$ & $\begin{array}{l}0 \\
0\end{array}$ & $\begin{array}{l}4 \\
3\end{array}$ & $\begin{array}{l}3 \\
4\end{array}$ & $\begin{array}{l}0 \\
0\end{array}$ & $\begin{array}{l}0 \\
1\end{array}$ & $\begin{array}{l}0 \\
0\end{array}$ & $\begin{array}{l}7 \\
8\end{array}$ & $\begin{array}{r}14 \\
8\end{array}$ \\
\hline MB8 & $\begin{array}{l}50 \\
50\end{array}$ & $\begin{array}{l}0 \\
0\end{array}$ & $\begin{array}{l}\mathbf{0} \\
\mathbf{0}\end{array}$ & $\begin{array}{l}2 \\
0\end{array}$ & $\begin{array}{l}19 \\
23\end{array}$ & $\begin{array}{l}8 \\
5\end{array}$ & $\begin{array}{l}1 \\
0\end{array}$ & $\begin{array}{l}0 \\
1\end{array}$ & $\begin{array}{l}1 \\
0\end{array}$ & $\begin{array}{l}31 \\
29\end{array}$ & $\begin{array}{l}39 \\
36\end{array}$ \\
\hline FAHET5 & 50 & 0 & 0 & 0 & 4 & 0 & 0 & 0 & 0 & 4 & 8 \\
\hline FAHET8 & 50 & 0 & 0 & 0 & 1 & 0 & 0 & 0 & 0 & 1 & 2 \\
\hline FAHET3 & 50 & 0 & 0 & 1 & 1 & 0 & 0 & 0 & 0 & 2 & 4 \\
\hline $\begin{array}{l}\text { Controls } \\
\text { ( } 9 \text { subjects) }\end{array}$ & 1150 & 0 & 1 & 2 & 28 & 6 & 4 & 0 & 0 & $\begin{array}{l}41 \\
\text { Mean } / 50 \text { cells } 1 \cdot 78 \\
\text { Range } 0-4\end{array}$ & $\begin{array}{l}\bar{M} \\
\text { Mean 3.22 } \\
\text { Range 0-8 }\end{array}$ \\
\hline
\end{tabular}

Variation was observed between the levels of MMC induced chromosome aberrations in repeated blood samples from individual patients. Three blood samples from FA3BI were treated with $0.1 \mu \mathrm{g} \mathrm{ml}^{-1}$ MMC resulting in 60,30 , and 30 induced aberrations per 50 cells respectively (table 3 ). FA9BI's lymphocytes were also treated with $0.1 \mu \mathrm{g} \mathrm{ml}^{-1}$ MMC on three separate occasions resulting in 133,60 , and 43 induced aberrations per 50 cells (table 3). Treatment with higher MMC doses resulted in less intersample variation (table 4), suggesting that at these doses the frequency of chromosome aberrations induced may have approached the maximum level possible before cell division was inhibited. Owing to the variation observed between different blood samples, comparison between results for different patients may not be valid. However, in one experiment blood samples from FA3BI and FA9BI were cultured and MMC treated simultaneously allowing a direct comparison of the results. Following treatment with 0.1 or $0.25 \mu \mathrm{g} \mathrm{ml}^{-1} \mathrm{MMC}$, lymphocytes from patient FA3BI contained 30 and 78 induced aberrations per
50 cells respectively, while lymphocytes from patient FA9BI contained 60 and 100 induced aberrations per 50 cells, suggesting that perhaps lymphocytes from patient FA9BI were more sensitive to MMC than those from FA3BI.

Treatment with $0.01 \mu \mathrm{g} \mathrm{ml}^{-1}$ DEB induced very few chromosome aberrations in lymphocytes from any of the persons studied. Results from six normal controls showed 0 to 1 induced aberrations per 50 cells (table 5). No aberrations were induced in lymphocytes from two obligate Fanconi's anaemia heterozygotes (FAHET3 and FAHET8). Results from patients ranged from 0 to 7 induced aberrations per 50 cells and the highest level of induced chromosome breakage was observed in lymphocytes from the asymptomatic patient MB8 (nine induced aberrations per 50 cells). Since the actual numbers of aberrations induced by $0.01 \mu \mathrm{g} / \mathrm{ml}^{-1}$ DEB were very small it is difficult to attribute any significance to apparent differences between results for different subjects. Treatment with a higher dose of DEB $\left(0 \cdot 1 \mu \mathrm{g} \mathrm{ml}^{-1}\right)$ also induced little chromosome breakage in normal lymphocytes $(0$ to 2 induced 
TABLE 3 Chromosome breakage following treatment with $0.1 \mathrm{\mu g} \mathrm{ml^{-1 }} \mathrm{MMC}$ (50 cells).

\begin{tabular}{|c|c|c|c|c|c|c|c|c|c|c|c|}
\hline \multirow[t]{2}{*}{ Subject } & \multicolumn{8}{|c|}{ No of } & \multirow{2}{*}{$\begin{array}{l}\text { Total No of } \\
\text { aberrations }\end{array}$} & \multirow{2}{*}{$\begin{array}{l}\% \text { aberrant } \\
\text { cells }\end{array}$} & \multirow{2}{*}{$\begin{array}{l}\text { No of aberrations } \\
\text { induced }\end{array}$} \\
\hline & $r$ & dic & $f$ & $c t g$ & $c t b$ & $\operatorname{csg}$ & $q r$ & $t r$ & & & \\
\hline FA2BI & 3 & 6 & 8 & 22 & 11 & 7 & 5 & 4 & 66 & 48 & 46 \\
\hline FA3BI & $\begin{array}{l}0 \\
0 \\
0\end{array}$ & $\begin{array}{l}2 \\
0 \\
0\end{array}$ & $\begin{array}{l}6 \\
0 \\
2\end{array}$ & $\begin{array}{l}26 \\
24 \\
18\end{array}$ & $\begin{array}{l}21 \\
11 \\
14\end{array}$ & $\begin{array}{l}0 \\
0 \\
1\end{array}$ & $\begin{array}{r}10 \\
3 \\
6\end{array}$ & $\begin{array}{r}10 \\
2 \\
1\end{array}$ & $\begin{array}{l}75 \\
40 \\
42\end{array}$ & $\begin{array}{l}66 \\
52 \\
54\end{array}$ & $\begin{array}{l}60 \\
30 \\
30\end{array}$ \\
\hline FA5BI & 0 & 0 & 6 & 16 & 19 & 0 & 1 & 0 & 42 & 40 & 19 \\
\hline FA6BI & 0 & 0 & 0 & 54 & 12 & 1 & 4 & 7 & 78 & 76 & 60 \\
\hline FA7BI & 0 & 0 & 9 & 46 & 17 & 2 & 6 & 1 & 81 & 66 & 54 \\
\hline FA8BI & 0 & 0 & 3 & 31 & 15 & 1 & 4 & 2 & 56 & 62 & 30 \\
\hline FA9BI & $\begin{array}{l}0 \\
0 \\
0\end{array}$ & $\begin{array}{l}0 \\
0 \\
0\end{array}$ & $\begin{array}{l}5 \\
1 \\
3\end{array}$ & $\begin{array}{r}101 \\
51 \\
43\end{array}$ & $\begin{array}{l}40 \\
39 \\
29\end{array}$ & $\begin{array}{l}0 \\
0 \\
0\end{array}$ & $\begin{array}{r}11 \\
2 \\
4\end{array}$ & $\begin{array}{l}3 \\
1 \\
3\end{array}$ & $\begin{array}{r}160 \\
94 \\
82\end{array}$ & $\begin{array}{l}88 \\
86 \\
80\end{array}$ & $\begin{array}{r}133 \\
60 \\
43\end{array}$ \\
\hline FA11BI & 0 & 0 & 1 & 21 & 17 & 2 & 5 & 0 & 46 & 56 & 38 \\
\hline MB8 & $\begin{array}{l}1 \\
0\end{array}$ & $\begin{array}{l}0 \\
1\end{array}$ & $\begin{array}{l}1 \\
6\end{array}$ & $\begin{array}{l}30 \\
68\end{array}$ & $\begin{array}{l}40 \\
31\end{array}$ & $\begin{array}{l}0 \\
6\end{array}$ & $\begin{array}{l}2 \\
3\end{array}$ & $\begin{array}{l}1 \\
1\end{array}$ & $\begin{array}{r}75 \\
116\end{array}$ & $\begin{array}{l}68 \\
68\end{array}$ & $\begin{array}{l}44 \\
87\end{array}$ \\
\hline FAHET3 & 0 & 0 & 1 & 3 & 1 & 0 & 0 & 0 & 5 & 10 & 3 \\
\hline FAHET5 & 0 & 1 & 2 & 3 & 0 & 0 & 1 & 0 & 7 & 12 & 3 \\
\hline FAHET8 & 0 & 0 & 0 & 1 & 0 & 1 & 0 & 0 & 2 & 4 & 1 \\
\hline $\begin{array}{l}\text { Controls } \\
\text { (600 cells, } \\
7 \text { subjects) }\end{array}$ & 0 & 1 & 5 & 12 & 5 & 0 & 1 & 1 & $\begin{array}{l}25 \\
\text { Mean } / 50 \text { cells } 2 \cdot 17 \\
\text { Range } 0-5\end{array}$ & $\begin{array}{l}\text { Mean 3.33 } \\
\text { Range 0-8 }\end{array}$ & $\begin{array}{l}\text { Mean } / 50 \text { cells } 0.58 \\
\text { Range } 0-4\end{array}$ \\
\hline
\end{tabular}

TABLE 4 Chromosome breakage following treatment with 0.25 or $0.5 \mu \mathrm{g} \mathrm{ml}^{-1} \mathrm{MMC}(50$ cells).

\begin{tabular}{|c|c|c|c|c|c|c|c|c|c|c|c|}
\hline \multirow[t]{2}{*}{ Subject } & \multicolumn{8}{|c|}{ No of } & \multirow{2}{*}{$\begin{array}{l}\text { Total No of } \\
\text { aberrations }\end{array}$} & \multirow{2}{*}{$\begin{array}{l}\% \text { aberrant } \\
\text { cells }\end{array}$} & \multirow{2}{*}{$\begin{array}{l}\text { No of aberrations } \\
\text { induced }\end{array}$} \\
\hline & $r$ & dic & $f$ & $c t g$ & $c t b$ & $\operatorname{csg}$ & $q r$ & $t r$ & & & \\
\hline $\begin{array}{l}0.25 \mu \mathrm{g} \mathrm{ml}^{-1} \\
\text { FA2BI }\end{array}$ & 0 & 2 & 4 & 29 & 13 & 6 & 5 & 8 & 67 & 52 & 47 \\
\hline $\begin{array}{l}\text { FA3BI } \\
\quad(25 \text { cells })\end{array}$ & $\begin{array}{l}0 \\
0\end{array}$ & $\begin{array}{l}1 \\
0\end{array}$ & $\begin{array}{l}7 \\
1\end{array}$ & $\begin{array}{l}27 \\
18\end{array}$ & $\begin{array}{l}35 \\
20\end{array}$ & $\begin{array}{l}1 \\
0\end{array}$ & $\begin{array}{l}21 \\
10\end{array}$ & $\begin{array}{r}19 \\
2\end{array}$ & $\begin{array}{r}111 \\
51\end{array}$ & $\begin{array}{l}82 \\
84\end{array}$ & $\begin{array}{l}96 \\
45\end{array}$ \\
\hline FA9BI & $\begin{array}{l}\mathbf{0} \\
\mathbf{0}\end{array}$ & $\begin{array}{l}\mathbf{0} \\
\mathbf{0}\end{array}$ & $\begin{array}{r}5 \\
10\end{array}$ & $\begin{array}{l}68 \\
69\end{array}$ & $\begin{array}{l}52 \\
44\end{array}$ & $\begin{array}{l}3 \\
2\end{array}$ & $\begin{array}{r}4 \\
10\end{array}$ & $\begin{array}{l}2 \\
2\end{array}$ & $\begin{array}{l}134 \\
137\end{array}$ & $\begin{array}{l}88 \\
82\end{array}$ & $\begin{array}{r}100 \\
98\end{array}$ \\
\hline FA11BI & & & 4 & 27 & 20 & & 12 & 4 & 67 & 62 & 59 \\
\hline $\begin{array}{l}\text { Controls } \\
\text { (200 cells, } \\
3 \text { subjects) }\end{array}$ & & & & & & & & & $\begin{array}{l}\text { Mean 3.75 } \\
\text { Range 2-7 }\end{array}$ & $\begin{array}{l}7 \\
4-12\end{array}$ & $\begin{array}{l}2 \\
0-5\end{array}$ \\
\hline $\begin{array}{l}0.5 \mu \mathrm{g} \mathrm{ml}^{-1} \\
\text { FA3BI }\end{array}$ & $\begin{array}{l}1 \\
0 \\
0\end{array}$ & $\begin{array}{l}3 \\
2 \\
0\end{array}$ & $\begin{array}{r}4 \\
22 \\
14\end{array}$ & $\begin{array}{l}61 \\
80 \\
81\end{array}$ & $\begin{array}{l}89 \\
92 \\
71\end{array}$ & $\begin{array}{l}3 \\
3 \\
2\end{array}$ & $\begin{array}{l}39 \\
49 \\
66\end{array}$ & $\begin{array}{l}35 \\
25 \\
37\end{array}$ & $\begin{array}{l}235 \\
273 \\
271\end{array}$ & $\begin{array}{l}98 \\
94 \\
98\end{array}$ & $\begin{array}{l}220 \\
263 \\
265\end{array}$ \\
\hline PA6BI & $\begin{array}{l}4 \\
0\end{array}$ & $\begin{array}{l}1 \\
0\end{array}$ & $\begin{array}{l}6 \\
6\end{array}$ & $\begin{array}{l}84 \\
67\end{array}$ & $\begin{array}{l}52 \\
61\end{array}$ & $\begin{array}{l}6 \\
3\end{array}$ & $\begin{array}{l}36 \\
23\end{array}$ & $\begin{array}{l}19 \\
11\end{array}$ & $\begin{array}{l}204 \\
171\end{array}$ & $\begin{array}{l}94 \\
84\end{array}$ & $\begin{array}{l}186 \\
151\end{array}$ \\
\hline FA10BI & 0 & 0 & 0 & 0 & 0 & 0 & 0 & 0 & 0 & 0 & 0 \\
\hline FA11BI & 0 & 0 & 4 & 33 & 48 & 3 & 17 & 14 & 119 & 74 & 112 \\
\hline FAHET3 & 0 & 0 & 0 & 6 & 0 & 0 & 0 & 0 & 6 & 8 & 4 \\
\hline FAHET5 & 0 & 0 & 2 & 10 & 3 & 2 & 4 & 0 & 21 & 38 & 17 \\
\hline FAHET8 & 0 & 0 & 0 & 5 & 1 & 0 & 0 & 0 & 6 & 12 & 5 \\
\hline $\begin{array}{l}\text { Controls } \\
\text { (650 cells, } \\
7 \text { subjects) }\end{array}$ & 0 & 0 & 4 & 54 & 17 & 0 & 12 & 0 & $\begin{array}{l}\text { Mean 6.7 } \\
\text { Range 0-18 }\end{array}$ & $\begin{array}{c}11 \cdot 8 \\
0-28\end{array}$ & $\begin{array}{l}5 \cdot 2 \\
0-17\end{array}$ \\
\hline
\end{tabular}


TABLE 5 Chromosome breakage following treatment with DEB (50 cells).

\begin{tabular}{|c|c|c|c|c|c|c|c|c|c|c|c|}
\hline \multirow[t]{2}{*}{ Subject } & \multicolumn{8}{|c|}{ No of } & \multirow{2}{*}{$\begin{array}{l}\text { Total No of } \\
\text { aberrations }\end{array}$} & \multirow{2}{*}{$\begin{array}{l}\% \text { aberrant } \\
\text { cells }\end{array}$} & \multirow{2}{*}{$\begin{array}{l}\text { No of aberrations } \\
\text { induced }\end{array}$} \\
\hline & $\boldsymbol{r}$ & dic & $f$ & ctg & $c t b$ & csg & $q r$ & $t r$ & & & \\
\hline \multicolumn{12}{|l|}{$0.01 \mu \mathrm{g} \mathrm{ml}^{-1}$} \\
\hline FA3BI & 0 & 0 & 2 & 8 & 5 & 0 & 2 & 0 & 17 & 26 & 3 \\
\hline FA7BI & 0 & 0 & 3 & 11 & 5 & 0 & $\mathbf{0}$ & 1 & 20 & 32 & 0 \\
\hline FA8BI & 0 & 0 & 1 & 5 & 8 & 0 & 0 & 0 & 14 & 18 & 0 \\
\hline FA9BI & 0 & 0 & 1 & 20 & 12 & 0 & 1 & 0 & 34 & 46 & 7 \\
\hline FAHET3 & 0 & 0 & 1 & 0 & 0 & 0 & 0 & 0 & 1 & 2 & 0 \\
\hline FAHET8 & 0 & 0 & 0 & 0 & 0 & 0 & 0 & 0 & 0 & 0 & 0 \\
\hline MB8 & 0 & 0 & 1 & 21 & 5 & 0 & 0 & 2 & 29 & 36 & 9 \\
\hline $\begin{array}{l}\text { Controls } \\
\text { (400 cells, } \\
6 \text { subjects) }\end{array}$ & 0 & 0 & 0 & 5 & 1 & 0 & 0 & 0 & $\begin{array}{l}\text { Mean 0.75 } \\
\text { Range 0-3 }\end{array}$ & $\begin{array}{l}1 \cdot 5 \\
0-6\end{array}$ & $\begin{array}{l}0 \cdot 125 \\
0-1\end{array}$ \\
\hline \multicolumn{12}{|l|}{$0.1 \mu \mathrm{g} \mathrm{ml}^{-1}$} \\
\hline FA3BI & 0 & 1 & 12 & 41 & 29 & 0 & 13 & 10 & 106 & 82 & 92 \\
\hline & 0 & 0 & 5 & 18 & 6 & 1 & 5 & 2 & 37 & 52 & 31 \\
\hline FA9BI & 0 & 0 & 13 & 114 & 56 & 0 & 8 & 1 & 192 & 90 & 165 \\
\hline FAHET3 & 0 & 0 & 5 & 3 & 3 & 0 & 0 & 0 & 11 & 16 & 9 \\
\hline FAHET8 & 0 & 0 & 0 & 0 & 0 & 0 & 0 & 0 & 0 & 0 & 0 \\
\hline MB8 & 0 & 0 & 8 & 59 & 20 & 0 & 1 & 3 & 91 & 74 & 71 \\
\hline Controls & 0 & 0 & 3 & 9 & 0 & 0 & 1 & 0 & Mean 1.9 & $3 \cdot 7$ & 0.4 \\
\hline $\begin{array}{l}\text { (350 cells, } \\
4 \text { subjects) }\end{array}$ & & & & & & & & & Range 0-6 & $0-12$ & $0-2$ \\
\hline
\end{tabular}

aberrations per 50 cells), and results for FAHET8 fell within the normal range. Lymphocytes from FAHET3 contained slightly more DEB induced aberrations at this dose (nine induced aberrations per 50 cells), but again the actual number of aberrations induced was small. Higher levels of DEB induced aberrations were observed in lymphocytes from FA3BI, FA9BI, and MB8. DEB treatment of two separate blood samples from FA3BI yielded rather different results, although in both cases the number of aberrations induced was abnormally high at 31 and 92 induced aberrations per 50 cells respectively (table 5 ).

DRUG THERAPY AND

CHROMOSOME ABERRATIONS

Drug therapy received by patients may affect chromosome instability. Six of the patients included in this study had received steroid therapy at some time (table 1) although only three (FA7BI, FA9BI, and FA10BI) were receiving such treatment at the time the blood samples were taken. Since no chromosome breakage was present in FA10BI's cells and high levels of breakage were seen in cells from untreated patients (FA6BI, FA8BI, and MB8), it seems unlikely that steroid therapy had any major effects on chromosome breakage.

\section{Discussion}

In the present study the greatly increased rates of spontaneous chromosome breakage in lymphocytes from six of the patients examined (FA2BI, FA5BI,
FA6BI, FA7BI, FA8BI, and FA9BI) were consistent with the clinical diagnosis of Fanconi's anaemia. Spontaneous aberration levels, however, s for FA3BI and FA11BI were only slightly above the normal range. Treatment of lymphocytes from all of $\stackrel{0}{\circ}$ these patients with either mitomycin $C$ or diepoxy- $\stackrel{\square}{\varrho}$ butane resulted in much higher levels of induced $\overrightarrow{\vec{B}}$ chromosome damage compared with normal con- $\frac{3}{3}$ trols. Contrary to earlier reports that the rate of $\supset$ spontaneous chromosome breakage in lymphocytes from Fanconi's anaemia patients fluctuates with time, ${ }^{6} 12$ consistent results were obtained here for 0 individual patients over a 2 year period. Similar results were reported by Cohen et al, ${ }^{7}$ suggesting 3 . that each patient may show a characteristic rate of 0 spontaneous lymphocyte chromosome breakage.

Patient MB8 with short stature and ptosis of the $\frac{\text { ? }}{3}$ right eyelid showed high levels of both spontaneous $D$ and mitomycin induced lymphocyte chromosome breakage. These results are clearly consistent with a diagnosis of Fanconi's anaemia. Since, however, she $\Omega$ has no anaemia, dysmorphic features are minimal, $\mathbb{O}$ and none of her three sibs is affected, a clinical diagnosis of Fanconi's anaemia cannot be made. It $O$ is possible that this patient has a disorder unrelated to Fanconi's anaemia, but we believe she has an atypical, clinically asymptomatic form of the disease. $\stackrel{\text { ? }}{+}$ This case is not unique as patients have previously $\square$ been described in whom the unusual chromosome breakage has been demonstrated before the presenta- $\stackrel{D}{\Omega}$ tion of the clinical symptoms early in adulthood. ${ }^{6}{ }^{13} \overrightarrow{\mathrm{D}}$

Surprisingly, patient FA10BI, who was referred $\bar{\sigma}$ as having some clinical features of Fanconi's 
anaemia, did not show increased levels of either spontaneous or drug induced chromosome abnormalities. This child had a short radius with failure of development of the distal third, giving a club hand deformity and little muscular control of the right thumb. No deformity was seen in the left hand. He had a normal platelet level, but required monthly blood transfusions for red cell aplasia and neutropenia. This patient does not show the typical clinical or chromosomal features of Fanconi's anaemia and we believe he may have a different genetic disorder. Interestingly, Cohen et $\mathrm{l}^{7}$ described a male child (RC) with red cell aplasia, neutropenia, and a virtually normal platelet count, but with some congenital abnormalities often associated with Fanconi's anaemia. Again, however, there was no evidence in their patient of unusual levels of spontaneous or drug induced chromosome aberrations in vitro. There are obviously similarities between this patient and FA10BI.

Results of DEB induced chromosome breakage in lymphocytes from two Fanconi's anaemia heterozygotes (table 5) support the assertion by Cohen et $\mathrm{al}^{7}$ that gene carriers cannot be detected consistently by their unusual response to this drug.

Overall, our results reaffirm the range of clinical phenotypes seen in Fanconi's anaemia and highlight the difficulties in diagnosis of some of these patients.

We thank Drs $M$ J Tarlow, D A Curnock, D I $K$ Evans, E M Belton, F G H Hill, S G N Richardson, M L Lewis, and A C Kendall for obtaining blood samples for us from their patients. We would also thank the Cancer Research Campaign for continued support, Professor D G Harnden for reading our manuscript, and Deborah Williams for typing the manuscript.

\section{References}

1 Alter BP, Rappeport JM, Parkman R. The bone marrow failure syndromes. In: Nathan DG, Oski FA, eds. Hematology of infancy and childhood. Philadelphia: Saunders, 1981:168-249.

2 Swift M. Fanconi's anaemia in the genetics of neoplasia. Nature $1971 ; 230: 370-3$.

${ }^{3}$ Estren S, Dameshek W. Familial hypoplastic anemia of childhood. Am J Dis Child 1947;73:671-87.

4 Glanz A, Fraser FC. Spectrum of anomalies in Fanconi anaemia. J Med Genet 1982;19:412-6.

5 Schroeder TM, Tilgen D, Kruger J, Vogel F. Formal genetics of Fanconi's anaemia. Humangenetik 1976;32: 257-88.

6 Schroeder TM, Drings P, Billner P, Buchinger G. Clinical and cytogenetic observation during a six year period in an adult with Fanconi anaemia. Blut 1976;34:119-32.

7 Cohen MM, Simpson SJ, Honig GR, Maurer HS, Nicklas JW, Martin AO. The identification of Fanconi anemia genotypes by clastogenic stress. Am J Hum Genet 1982;34:794-810.

8 Sasaki MS, Tonomura A. A high susceptibility of Fanconi's anaemia to chromosome breakage by DNA cross linking agents. Cancer Res 1973;33:1829-36.

9 Auerbach AD, Wolman SR. Susceptibility of Fanconi's anaemia fibroblasts to chromosome damage by carcinogens. Nature 1976;261:494-6.

10 Sasaki MS. Fanconi anemia: a condition possibly associated with defective DNA repair. In: Hanawalt PC, Friedberg EC, Fox CF, eds. DNA repair mechanisms. New York: Academic Press, 1978: 675-84.

11 An international system for human cytogenetic nomenclature (ISCN). Cytogenet Cell Genet 1978;21:309-402.

12 Berger R, Bussel A, Schenmetzler C. Somatic segregation and Fanconi anaemia. Clin Genet 1977;11:409-15.

13 Schroeder TM, Pohler E, Hufnagl HD, Stahl-Mauge C. Fanconi's anaemia: terminal leukaemia and 'forme fruste' in one family. Clin Genet 1979;16:260-8.

Correspondence and requests for reprints to $\mathrm{Dr}$ G Duckworth-Rysiecki, Department of Genetics, The Hospital for Sick Children, 555 University Avenue, Toronto, Ontario, Canada M5G 1X8. 